\title{
Counting of apoptotic cells: a methodological study in invasive breast cancer
}

\author{
H Anna Maria van de Schepop, Johannes S de Jong, Paul J van Diest, Jan P A Baak
}

\begin{abstract}
Aims-To arrive at a reproducible sampling technique for counting apoptotic cells in tissue sections of invasive breast cancer that can serve as a protocol for further clinical studies.

Methods-In $4 \mu \mathrm{m}$ thick haematoxylin and eosin stained tissue sections of 12 breast cancers, apoptotic cells, recognised by strict morphological criteria, were counted in consecutive fields of vision at $\times 1000$ magnification in a marked area in the most poorly differentiated region of tumour. These counts were regarded as the gold standard. Subsequently, in a systematic sampling experiment, the number of fields that had to be counted to derive an acceptable coefficient of variation (CV) was determined. To compare counts at different magnifications, all fields of vision were also counted at $\times 630$ and $\times 400$. The intra- and inter-observer reproducibility was tested by repeated measurements at these magnifications in 10 systematically selected fields of vision.
\end{abstract}

Results-Apoptosis seemed to be a rare event, affecting, on average, about $1 \%$ of tumour cells. Noticeable clustering of apoptotic cells was observed. The systematic sampling experiment showed that at $\times 1000$ magnification, the $C V$ was improved by counting up to 20 fields. When comparing $\times 400$ and $\times 630$ magnifications with the $\times 1000$ magnification, the correlation coefficients were 0.88 and 0.87 , respectively. However, the lower magnifications yielded lower counts. With regard to reproducibility, the intra-observer correlation coefficient was 0.91 at $\times 630$ and 0.76 at $\times 400$. The inter-observer correlation coefficient was 0.77 at $\times 630$ and 0.68 at $\times 400$.

Conclusions-Apoptotic cells can be counted readily in haematoxylin and eosin stained tissue sections. However, a systematic sampling protocol must be followed and cells should be counted at a relatively high magnification to obtain acceptable reproducibility. The suggested protocol will permit further correlative and prognostic studies and the monitoring of the effects of treatment.

(f Clin Pathol: Mol Pathol 1996;49:M214-M217)

Pathology, Free University Hospital, PO Box 7057, NL-1007 MB Amsterdam, The Netherlands

Correspondence to: Dr P J van Diest.

Accepted for publication 18 June 1996
Breast cancer is the most common cancer

affecting women in the western world, the inci-
Keywords: breast cancer, apoptosis, sampling, reproducibility. dence of which is still increasing, especially among younger women. Despite radical surgery, many patients die of distant metastases. Therefore, adjuvant therapy is indicated in high risk patients. These patients are usually identified by lymph node positivity. However, the accuracy of lymph node status in predicting prognosis is only about $65 \%$, so additional prognostic factors are desirable. Proliferation variables assessed in the primary tumour are prognostically very important in breast cancer, ${ }^{1-13}$ probably because the proliferative capacity of established distant metastases can be predicted from the primary tumour. Tumour growth, however, is a balance between proliferation on the one hand and cell death by necrosis and apoptosis on the other. It is often claimed that apoptosis is the most important component of continuous cell loss in tumours, although quantitative data are as yet rare. ${ }^{14-16}$ Apoptosis is an important and interesting mechanism for cell death as it affects individual cells and is, more or less, an endogenous process. Expression of genes inhibiting ( $b c l-2)$ or promoting (p53, c-myc) apoptosis may be altered clonally in tumour cells by-for example, mutations or deletions, being the same in the primary tumour and its metastases. One study ${ }^{17}$ has, in this context, showed apoptotic counts to be prognostically important. Reliable methods for assessing the number of apoptotic cells are of the utmost importance for the study of prognosis and cell biology.

Several methods have been applied to assess apoptosis. Flow cytometry has been used ${ }^{18-22}$ and markers for apoptotic cells have been described which may facilitate counting. ${ }^{23}$ Apoptotic cells, however, can be easily recognised in haematoxylin and eosin stained tissue sections. ${ }^{17}{ }^{23}$ However, methodological studies evaluating sources of error in the enumeration of apoptotic cells are rare. The aim of this study, therefore, was to develop a reproducible sampling protocol for counting apoptotic cells in haematoxylin and eosin stained sections. Such a protocol could be used to monitor the effects of treatment induced apoptosis.

\section{Methods}

Haematoxylin and eosin stained sections, $4 \mu \mathrm{m}$ thick, from 12 randomly selected cases of preoperatively untreated invasive ductal breast cancer were studied. All tumour material had been cut into slices $0.5 \mathrm{~cm}$ thick and fixed in neutral buffered formalin for 12-24 hours. In these sections, the most poorly differentiated invasive area at the periphery of the tumour was marked for counting of apoptotic cells (fig 1). 
Table 1 Morphological criteria for apoptotic cells

Retracted, often pink to orange cytoplasm

Condensed nuclear chromatin

Earliest stage shows condensation of chromatin along the nuclear membrane, later forming uniformly condensed to round/oval, dark structures and then breaking up into several small, round, dark fragments

Concerns isolated cells

No inflammatory reaction

Cells were recognised as apoptotic according to the strict morphological criteria listed in table 1 .

In the first experiment, apoptotic cells were counted twice by the same observer (HvdS) in all fields of vision (up to a maximum of 100) containing invasive tumour at a magnification of $\times 1000$ (objective $\times 100$, field diameter 180 $\mu \mathrm{m})$. The correlation coefficient between the

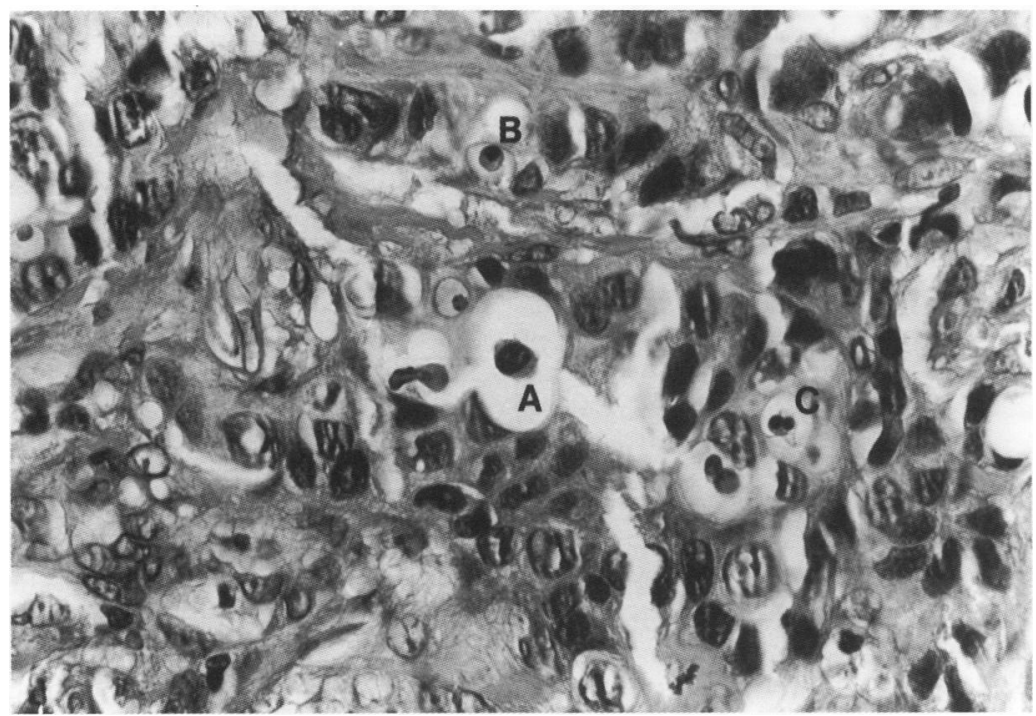

Figure 1 Examples of apoptotic cells. Early phase apoptosis $(A)$ with condensed chromatin along the nuclear border and retracted cytoplasm. Late phase apoptosis (B) showing homogeneously dark single nuclear remnants and retracted cytoplasm. An apoptotic cell $(C)$ with nuclear fragmentation and retracted cytoplasm.

\begin{tabular}{|l|l|l|l|l|l|l|l|l|l|}
\hline 0 & 1 & 0 & 0 & 1 & 1 & 1 & 0 & 0 & 0 \\
\hline 1 & 1 & 0 & 0 & 0 & 0 & 0 & 0 & 1 & 0 \\
\hline 0 & 0 & 2 & 2 & 0 & 1 & 0 & 1 & 0 & 0 \\
\hline 1 & 0 & 1 & 1 & 0 & 0 & 1 & 1 & 0 & 2 \\
\hline 0 & 0 & 0 & 0 & 1 & 0 & 2 & 1 & 0 & 0 \\
\hline 0 & 2 & 0 & 1 & 0 & 0 & 1 & 0 & 0 & 0 \\
\hline 0 & 0 & 0 & 0 & 1 & 2 & 0 & 0 & 0 & 1 \\
\hline 0 & 1 & 0 & 0 & 0 & 0 & 1 & 0 & 2 & 0 \\
\hline 0 & 0 & 1 & 1 & 0 & 2 & 0 & 0 & 0 & 0 \\
\hline 0 & 0 & 2 & 0 & 1 & 0 & 0 & 1 & 1 & 0 \\
\hline
\end{tabular}

Figure 2 Example of an "apoptotic map". Each box represents one field of vision at $\times 1000\left(0.0255 \mathrm{~mm}^{2}\right)$. Note the clustering: a single apoptotic figure is present in the box composed of eight fields at the bottom left. In contrast, in the box of eight fields at the top right, seven apoptotic figures are present. first and second assessments was 0.99 . These counts were then used as a gold standard. For each case, a map was composed showing 100 fields with the apoptotic count depicted per field, and referred to as the "apoptotic map" (fig 2).

Using these data, a sampling simulation experiment was carried out based on systematic sampling. ${ }^{24}$ The latter principle ensures that fields of vision selected for counting are evenly spread over the measurement area. For a sample size of two, 50 systematic samples can be drawn: fields $1 / 51,2 / 52,3 / 53, \ldots 50 / 100$. When the counts of these samples differ greatly, this sample size does not yield reproducible results. The difference between the samples is expressed by the variation around the mean of these 50 samples (coefficient of variation $(\mathrm{CV}) ; 100 \% \times \mathrm{SD} /$ mean $)$. One would expect the $\mathrm{CV}$ to decrease with increasing sample size. Therefore, the sampling procedure was repeated for a sample size of three (fields $1 / 34 / 67,2 / 35 / 68, \ldots 33 / 66 / 100$ ) to a sample size of 50 . For each sample size, the $C V$ was calculated as described above. The results for the 12 cases were depicted in a graph showing the relation between sample size and $\mathrm{CV}$ for each case.

In a second experiment, the accuracy of apoptotic cell counting at different magnifications was assessed by counting all fields of vision in the area marked at $\times 400$ (field diameter $445 \mu \mathrm{m}$ ) and $\times 630$ (field diameter $275 \mu \mathrm{m}$ ) and by comparing these counts with the values obtained at $\times 1000$. The intra- and interobserver reproducibility of the apoptotic counts at the former two magnifications was tested by counting apoptotic cells, in duplicate, in 10 randomly sampled fields of vision at an interval of at least one week by the same observer (HvdS) and different observers (HvdS, JdJ). Linear regression analysis was performed on the paired data using the Statistical Package for the Social Sciences (SPSS, Chicago, Illinois, USA), registering the correlation coefficient $R$.

\section{Results}

In most cases the apoptotic maps showed a noticeable clustering of apoptotic cells. An example of an apoptotic map, illustrating this clustering, is shown in fig $2(\times 1000)$. Each box measures $0.0255 \mathrm{~mm}^{2}$ at the specimen level, with an average number of 75 nuclei per box. A single apoptotic cell is present in the box of eight fields of vision at the bottom left, whereas seven apoptotic cells are present in a box of similar size and shape at the top right of the figure.

Figure 3 shows the relation between sample size and $\mathrm{CV}$ from the systematic sampling experiment. Up to a sample of 20 fields, the CV gradually decreased, but assessing more fields of vision did not improve these results greatly.

Table 2 shows, for each of the 12 cases, a comparison of the sum of the apoptotic counts of all fields at different magnifications, all data normalised to $\mathrm{mm}^{2}$. The apoptotic counts ranged from 3 to 94 per $\mathrm{mm}^{2}$ at $\times 400$, from 5 to 
$155 \mathrm{~mm}^{2}$ at $\times 630$ and from 7 to 269 per $\mathrm{mm}^{2}$ at $\times 1000$. Overall, the average apoptotic count decreased from 47 at $\times 1000$ to 27 at $\times 630$ and 16 at $\times 400$. Furthermore, when comparing counts in individual cases, lower magnifications yielded lower counts. When comparing (linear regression analysis) the $\times 400$ and $\times 630$ magnifications with the $\times 1000$ magnification,

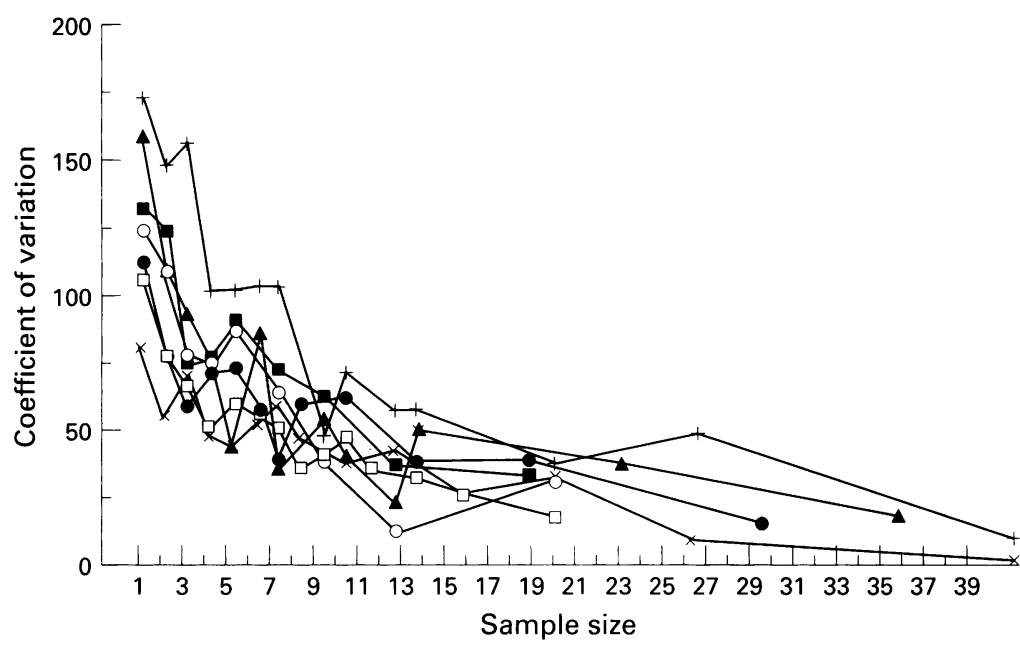

Figure 3 Relation between sample size and $C V$ in a systematic sampling experiment for the apoptotic count.

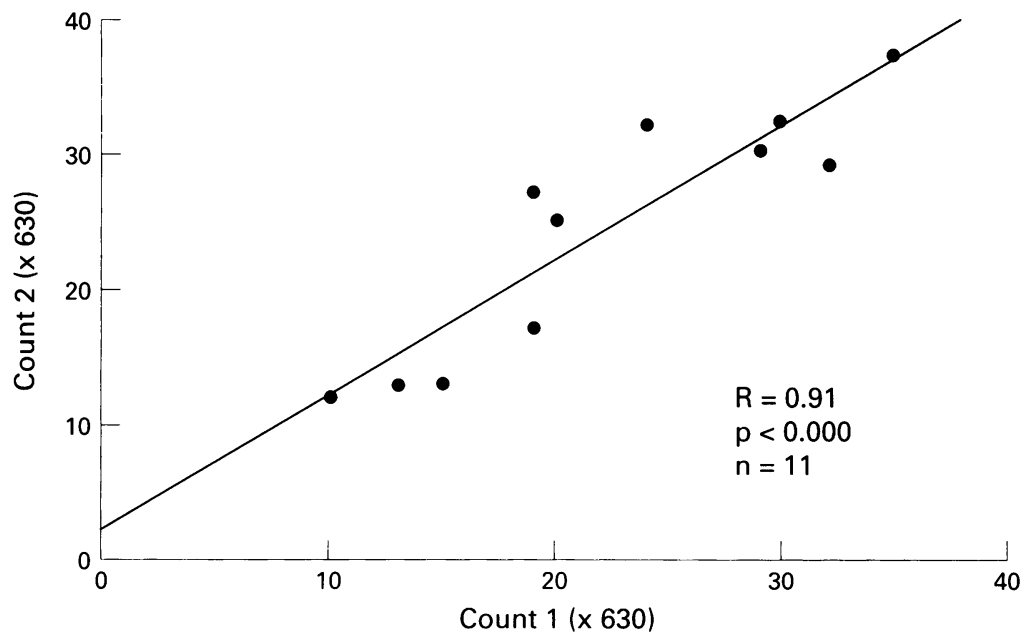

Figure 4 Intra-observer reproducibility of the apoptotic counts at $\times 630$ (systematic random sampling).

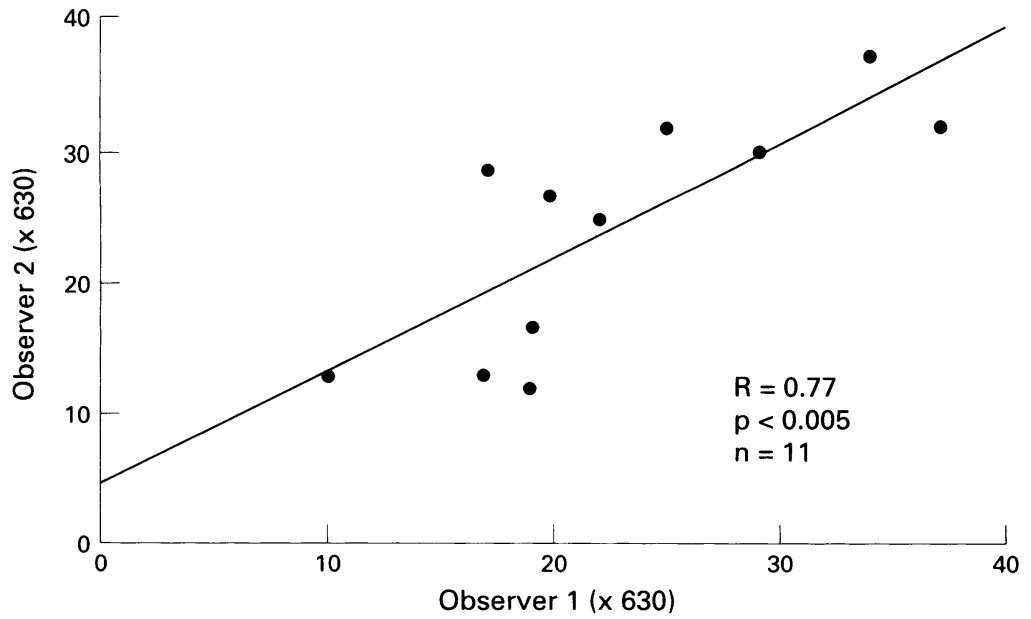

Figure 5 Inter-observer reproducibility of apoptotic counts at $\times 630$ (systematic random sampling).
Table 2 Apoptotic counts at $\times 400, \times 630$ and $\times 1000$ in 12 cases of invasive breast cancer (expressed per $\mathrm{mm}^{2}$ )

\begin{tabular}{lccc}
\hline & \multicolumn{3}{c}{ Magnification } \\
\cline { 2 - 4 } Case number & $\times 400$ & $\times 630$ & $\times 1000$ \\
\hline 1 & 4 & 6 & 10 \\
2 & 6 & 7 & 14 \\
3 & 6 & 13 & 20 \\
4 & 9 & 10 & 12 \\
5 & 4 & 10 & 19 \\
6 & 94 & 155 & 269 \\
7 & 18 & 25 & 69 \\
8 & 14 & 31 & 58 \\
9 & 8 & 23 & 26 \\
10 & 12 & 18 & 22 \\
11 & 14 & 25 & 37 \\
12 & 3 & 5 & 7 \\
Average & 16 & 27 & 47 \\
\hline
\end{tabular}

Table 3 Intra- and inter-observer correlation coefficients of apoptotic counts at different magnifications in 11 cases of invasive breast cancer (case 6 excluded; see text)

\begin{tabular}{lll}
\hline & \multicolumn{2}{l}{ Correlation coefficient } \\
\cline { 2 - 3 } Magnification & Intra-observer & Inter-observer \\
\hline$\times 400$ & 0.76 & 0.68 \\
$\times 630$ & 0.91 & 0.77 \\
\hline
\end{tabular}

the correlation coefficients were 0.88 (slope $=0.22)$ and $0.87($ slope $=0.39)$, respectively.

Table 3 shows the results of the intra- and inter-observer reproducibility experiments. For these experiments, case 6 (which had a very high apoptotic count) was excluded from the analysis as this outlier biased the results positively. The intra-observer correlation coefficient was 0.91 at $\times 630$ and 0.76 at $\times 400$. The inter-observer correlation coefficients were 0.77 and 0.68 at $\times 630$ and $\times 400$, respectively. Figures 4 and 5 show the results of the intraand inter-observer reproducibility tests at $\times 630$.

\section{Discussion}

The aim of the present study was to arrive at a reproducible sampling technique for counting apoptotic cells in tissue sections of invasive breast cancer that can serve as a protocol for further clinical studies.

Apoptotic cells were easily recognisable in the haematoxylin and eosin stained sections Using strict criteria, they were readily distinguishable from mitoses and lymphocytes. We therefore feel that special stains, such as in situ end labelling, ${ }^{23}$ are not essential for the recognition of apoptotic cells.

Apoptosis seemed to be a relatively rare event in most of the tissue sections studied, with an average of 47 apoptotic figures per $\mathrm{mm}^{2}$. Assuming a cellularity of 5000 tumour cells per $\mathrm{mm}^{2}$, this corresponds to an average of about $1 \%$ of tumour cells. Obviously, in reality, a larger proportion of tumours cells will be undergoing apoptosis, as the morphological criteria listed in table 1 are not present throughout apoptosis. Furthermore, morphologically apparent apoptosis is present for just a few hours. ${ }^{25}$ The relative rarity of apoptosis suggests that flow cytometry, which is often applied to cell lines, ${ }^{18-22}$ is less suitable for 
studying apoptosis in human breast cancer and possibly also for other human tumours. In tissue sections, sampling is difficult not only because of the rarity of apoptosis but also because of the clustering phenomenon. Systematic sampling, which has been found to be useful for other applications, ${ }^{24}$ is therefore mandatory.

The $\times 1000$ magnification should be regarded as the gold standard, as small apoptotic figures present in the later phases of apoptosis can be recognised. However, because of the small field of vision, extensive sampling is required, and the use of an oil immersion lens is impractical. We therefore also tested lower magnifications. At $\times 630$, some apoptotic cells may be missed, as the counts at this magnification were systematically lower than at $\times 1000$. However, the intra- and inter-observer reproducibility was still good at $\times 630$, and sampling at this magnification takes less time and does not require the use of an oil immersion lens. At $\times 400$, the counts were, on average, even lower and reproducibility was noticeably worse. Therefore, when considering reproducibility, efficiency and practicality, a protocol based on random sampling at $\times 630$ seems optimal. One can accept the small systematic error resulting from the omission of some small apoptotic figures. This protocol can be used to follow large groups of patients with invasive breast cancer to evaluate the prognostic value of apoptotic counts compared with proliferation variables and to study correlations between apoptotic counts and other prognostic variables.

These results expand on those of Wijsman et $a l,{ }^{23}$ who found good reproducibility when counting apoptotic cells in whole tissue sections at $\times 630$. No reproducibility data were included in a previous clinical study. ${ }^{17} \mathrm{Com}-$ pared with flow cytometry, counting in sections has the advantage of being able to select a specific morphological area for counting (thus avoiding benign cells) and that it is also suitable for detecting a few apoptotic figures within numerous tumour cells.

In conclusion, apoptotic cells can be counted readily in haematoxylin and eosin stained tissue sections. However, a systematic sampling protocol must be followed and cells should be counted at a relatively high magnification to obtain acceptable reproducibility. The suggested protocol will permit further correlative and prognostic studies and the monitoring of the effects of treatment. Supported in part by grants 28-2015 and 28-1814 of the Prae-
ventiefonds.

1 Aaltomaa S, Lipponen P, Eskelinen M, Kosma VM, Marin $\mathrm{S}$, Alhava $\mathrm{E}$, et al. Prognostic factors in axillary lymph node-negative (pN-) breast carcinomas. Eur $\mathcal{f}$ Cancer 1991 27:1555-9.
2 Baak JPA, Dop H van, Kurver PHJ, Hermans J. The value of morphometry to classic prognosticators in breast cancer. Cancer 1985;56:374-82.

3 Bouzubar N, Walker KJ, Griffiths K, Ellis IO, Elston CW, Robertson JFR, et al. Ki67 immunostaining in primary breast cancer: pathological and clinical associations. $B r F$ Cancer 1989;59:943-7.

4 Clayton F. Pathologic correlates of survival in 378 lymph node-negative infiltrating ductal breast carcinomas. Mitotic count is the best single predictor. Cancer 1991; 68: $1309-17$.

5 Diest PJ van, Baak JPA. The morphometric multivariate prognostic index (MPI) is the strongest prognosticator in premenopausal lymph node negative and lymph node positive breast cancer patients. Hum Pathol 1992;22:479 89.

6 Joensuu H, Toikkanen S, Klemi PJ. DNA index and S-phase fraction and their combination as prognostic factors in operable ductal breast carcinoma. Cancer 1990;66:33140.

7 Kamel OW, Franklin WA, Ringus JC, Meyes JS. Thymidine labeling index and $\mathrm{Ki}-67$ growth fraction in lesions of the breast. Am $\mathcal{F}$ Pathol 1989;134:107-13.

8 Linden JC van der, Baak JPA, Lindeman J, Hermans J, Meijer CJLM. Prospective evaluation of prognostic value of morphometry in patients with primary breast cancer. $\mathcal{F}$ Clin Pathol 1987;40:302-6.

9 Meyer JS, Friedman E, McCrate MM, Bauer WC. Prediction of early course of breast carcinoma by thymidine labeling. Cancer 1983;51:1879-86.

10 Sahin AA, Ro J, Ro JY, Thick MB, El-Nagger AK, Ordone $\mathrm{NG}$, et al. Ki67 immunostaining in node-negative stage I/II breast carcinoma. Significant correlation with prognosis. Cancer 1991;68:549-57.

11 Sigurdsson H, Baldetorp B, Borg A, Dalberg M, Ferno M, Killander D, et al. Indicators of prognosis in node-negative breast cancer. $N$ Engl f Med 1990;322:1045-53.

12 Uyterlinde AM, Schipper NW, Baak JPA, Peterse H, Matze P. Limited prognostic value of cellular DNA content to classical and morphological parameters in invasive ducta breast cancer. Am $\mathcal{F}$ Clin Pathol 1988;89:301-7.

13 Weidner N, Moore DH, Ljung BM, Waldman FM, Goodson WH, Mayall B, et al. Correlation of bromodeoxyuridine (BrdU) labeling of breast carcinoma cells with mitotic figure content and tumor grade. Am $\mathcal{F}$ Surg Pathol mitotic figure con

14 Moore JV. Death of cells and necrosis of tumours. In: Potten CS, ed. Perspectives on mammalian cell death. Oxford: Oxford University Press, 1987:305.

15 Sarraf CE, Bowen ID. Proportions of mitotic and apoptotic cells in a range of untreated experimental tumours. Cell Tissue Kinet 1988;21:45-9.

16 Wyllie AH. Apoptosis and the regulation of cell numbers in normal and neoplastic tissues; an overview. Cancer Metastasis Rev 1992;11:95-103.

17 Lipponen P, Aaltoma S, Kosma VM, Syrjanen K. Apoptosis in breast cancer as related to histopathological characteristics and prognosis. Eur $\mathcal{F}$ Cancer 1994;30A:2068-73.

18 Afanasyev VN, Korol BA, Matylevich NP, Pechatnikow VA, Umansky SR. The use of flow cytometry for the investigation of cell death. Cytometry 1993;14:603-9.

19 Belloc F, Dumain P, Boisseau MR, Jalloustre C, Reiffers J, Bernard P, et al. A flow cytometric method using Hoechst 33342 and propidium iodide for simultaneous cell cycle analysis and apoptosis determination in unfixed cells. Cytometry 1994;17:59-65.

20 Nicoletti I, Migliorati G, Pagliacci MC, Grignani F, Riccardi C. A rapid and simple method for measuring thymocyte apoptosis by propidium iodide staining and flow cytometry. F Immunol Method 1991;139:271-9.

21 Swat W, Ignatowicz L, Kisielow P. Detection of apoptosis in immature $\mathrm{CD} 4+8+$ thymocytes by flow cytometry. $\mathcal{F}$ Immunol Method 1991;137:79-87.

22 Zamai L, Falcieri E, Zauli G, Cataldi A, Vitale M. Optima detection of apoptosis by flow cytometry depends on cell morphology. Cytometry 1993;14:891-7.

23 Wijsman JH, Jonker RR, Keijzer R, Velde CJH van de, Cornelisse CJ, Dierendonck JH van. A new method to detect apoptosis in paraffin sections: in situ end labeling of fragmented DNA. ₹ Histochem Cytochem 1993;41:7-12.

24 Fleege JC, Diest PJ van, Baak JPA. Computer assisted efficiency-testing of different sampling methods for
selective nuclear graphic tablet morphometry. Lab Invest 1990;63:270-5.

25 Bursch W, Paffe S, Putz B, Barthel G, Schulte-Hermann R. Determination of the length of the histological stages of apoptosis in normal liver and in altered hepatic foci of rats. Carcinogenesis 1990;11:847. 